\title{
2015 AUDITED SCHEDULE OF CHANGES IN NET ASSETS
}

\author{
DOI: http://dx.doi.org/10.3163/1536-5050.104.4.E1
}

The table below summarizes the association's financial status as of December 31, 2015. For a more complete audit report and related information, see the 2014/15 MLA Annual Report <http://www. mlanet.org/p/do/si/topic=219 (members only) $>$. This report includes balance sheets, fund status reports, budgeted and actual revenues and expenditures, and a schedule of investments. Members may obtain a copy of the audit report from MLA headquarters.

Additional information regarding MLA finances appears in the April 2016 issue of the MLA News.

\begin{tabular}{|c|c|c|c|c|c|c|}
\hline & $\begin{array}{l}\text { Net assets } \\
\text { January } 1 \text {, } \\
\quad 2015\end{array}$ & $\begin{array}{l}\text { Contributions } \\
\text { and other } \\
\text { revenue }\end{array}$ & $\begin{array}{l}\text { Investment } \\
\text { income }\end{array}$ & $\begin{array}{l}\text { Net assets } \\
\text { released from } \\
\text { restrictions }\end{array}$ & Expenses & $\begin{array}{c}\text { Net assets } \\
\text { (deficit) } \\
\text { December 31, } \\
2015\end{array}$ \\
\hline \multicolumn{7}{|l|}{ Unrestricted net assets } \\
\hline General operating & $\$(175,700)$ & $\$ 2,738,450$ & - & - & $\$(2,732,742)$ & $\$(169,992)$ \\
\hline Loss on disposal of property and equipment & - & - & - & - & $(53,476)$ & $(53,476)$ \\
\hline Total general operating & $(175,700)$ & $2,738,450$ & - & - & $(2,786,218)$ & $(223,468)$ \\
\hline \multicolumn{7}{|l|}{ Other funds: } \\
\hline Association stabilization & $1,487,423$ & - & $(25,514)$ & - & $(13,426)$ & $1,448,483$ \\
\hline Capital equipment & 2,228 & - & - & - & - & 2,228 \\
\hline Special purpose & - & - & - & 68,757 & $(68,757)$ & - \\
\hline Sections & 368,674 & 48,837 & - & - & $(53,175)$ & 364,336 \\
\hline Total unrestricted net assets & $\$ 1,858,325$ & $\$ 48,837$ & $\$(25,514)$ & $\$ 68,757$ & $\$(135,358)$ & $\$ 1,815,047$ \\
\hline \multicolumn{7}{|l|}{ Temporarily restricted net assets } \\
\hline $\begin{array}{l}\text { Ysabel Bertolucci MLA Annual Meeting Grant } \\
\text { Endowment }\end{array}$ & 946 & - & $(500)$ & $(1,000)$ & - & $(554)$ \\
\hline $\begin{array}{l}\text { Estelle Brodman Award for the Academic Medical } \\
\text { Librarian of the Year Endowment }\end{array}$ & 40,927 & - & (593) & $(500)$ & - & 39,834 \\
\hline $\begin{array}{l}\text { Naomi C. Broering Hispanic Heritage Grant } \\
\text { Endowment }\end{array}$ & - & - & (516) & - & - & $(516)$ \\
\hline $\begin{array}{l}\text { Lois Ann Colaianni Award for Excellence and } \\
\text { Achievement in Hospital Librarianship Endowment }\end{array}$ & 9,953 & - & (296) & $(500)$ & - & 9,157 \\
\hline $\begin{array}{l}\text { Cunningham Memorial International Fellowship } \\
\text { Endowment }\end{array}$ & 29,174 & - & $(2,489)$ & - & - & 26,685 \\
\hline $\begin{array}{l}\text { Louise Darling Medal for Distinguished Achievement in } \\
\text { Collection Development in the Health Sciences } \\
\text { Endowment }\end{array}$ & 42,308 & - & (732) & $(1,000)$ & - & 40,576 \\
\hline Janet Doe Lectureship Endowment & 30,202 & - & (523) & (250) & - & 29,429 \\
\hline $\begin{array}{l}\text { Carla J. Funk Governmental Relations Award } \\
\text { Endowment }\end{array}$ & 1,520 & - & (225) & (500) & - & 795 \\
\hline Eugene Garfield Research Fellowship Endowment & 1,656 & - & $(2,532)$ & $(5,000)$ & - & $(5,876)$ \\
\hline $\begin{array}{l}\text { T. Mark Hodges International Service Award } \\
\text { Endowment }\end{array}$ & 536 & - & $(97)$ & $(500)$ & - & $(61)$ \\
\hline $\begin{array}{l}\text { Hospital Libraries Section/MLA Professional } \\
\text { Development Grant Endowment }\end{array}$ & 18,584 & - & (560) & - & - & 18,024 \\
\hline David A. Kronick Traveling Fellowship Endowment & 1,222 & - & (529) & $(2,000)$ & - & $(1,307)$ \\
\hline Joseph Leiter NLM/MLA Lectureship Endowment & 30,519 & - & (836) & $(1,366)$ & - & 28,317 \\
\hline $\begin{array}{l}\text { Librarians without Borders }{ }^{\circledR} \text { Ursula Poland International } \\
\text { Scholarship Endowment }\end{array}$ & 3,248 & - & (455) & $(1,000)$ & - & 1,793 \\
\hline $\begin{array}{l}\text { Donald A. B. Lindberg Research Fellowship } \\
\text { Endowment }\end{array}$ & 71,144 & - & $(4,144)$ & - & - & 67,000 \\
\hline Majors/MLA Chapter Project of the Year Endowment & 7,715 & - & (233) & (500) & - & 6,982 \\
\hline $\begin{array}{l}\text { Lucretia W. McClure MLA Excellence in Education } \\
\text { Award Endowment }\end{array}$ & 11,946 & - & (680) & - & - & 11,266 \\
\hline John P. McGovern Award Lectureship Endowment & 7,093 & - & $(2,339)$ & $(5,000)$ & - & (246) \\
\hline MLA Disaster Relief Fund & 5,279 & 125 & - & - & - & 5,404 \\
\hline Scholarship Endowment & 8,058 & - & $(5,200)$ & $(15,799)$ & - & $(12,941)$ \\
\hline Section Project of the Year Award Endowment & 1,358 & - & - & $(500)$ & - & 858 \\
\hline Shaping Our Future Endowment & 9,575 & - & (977) & - & - & 8,598 \\
\hline Special Purpose/Historical Marker & 1,500 & 1,000 & - & $(2,500)$ & - & - \\
\hline Special Purpose/Librarians without Borders ${ }^{\circledR}$ & 1,053 & 52,000 & - & $(30,842)$ & - & 22,211 \\
\hline Special Purpose/MLA/NLM Spectrum Scholarships & 65,000 & - & - & - & - & 65,000 \\
\hline Totals, temporarily restricted net assets & $\$ 400,516$ & $\$ 53,125$ & $\$(24,456)$ & $\$(68,757)$ & - & $\$ 360,428$ \\
\hline
\end{tabular}

Table 1

Medical Library Association schedule of changes in net assets by fund year ended December 31, 2015 


\begin{tabular}{|c|c|c|c|c|c|c|}
\hline & $\begin{array}{l}\text { Net assets } \\
\text { January } 1 \text {, } \\
2015\end{array}$ & $\begin{array}{l}\text { Contributions } \\
\text { and other } \\
\text { revenue }\end{array}$ & $\begin{array}{l}\text { Investment } \\
\text { income }\end{array}$ & $\begin{array}{l}\text { Net assets } \\
\text { released from } \\
\text { restrictions }\end{array}$ & Expenses & $\begin{array}{l}\text { Net assets } \\
\text { (deficit) } \\
\text { December 31, } \\
2015\end{array}$ \\
\hline \multicolumn{7}{|l|}{ Permanently restricted net assets: } \\
\hline $\begin{array}{l}\text { Ysabel Bertolucci MLA Annual Meeting Grant } \\
\text { Endowment }\end{array}$ & 24,779 & 2,089 & - & - & - & 26,868 \\
\hline $\begin{array}{l}\text { Estelle Brodman Award for the Academic Medical } \\
\text { Librarian of the Year Endowment }\end{array}$ & 29,248 & - & - & - & - & 29,248 \\
\hline $\begin{array}{l}\text { Naomi C. Broering Hispanic Heritage Grant } \\
\text { Endowment }\end{array}$ & 25,493 & - & - & - & - & 25,493 \\
\hline $\begin{array}{l}\text { Lois Ann Colaianni Award for Excellence and } \\
\text { Achievement in Hospital Librarianship Endowment }\end{array}$ & 14,650 & 625 & - & - & - & 15,275 \\
\hline $\begin{array}{l}\text { Cunningham Memorial International Fellowship } \\
\text { Endowment }\end{array}$ & 122,971 & 50 & - & - & - & 123,021 \\
\hline $\begin{array}{l}\text { Louise Darling Medal for Distinguished Achievement in } \\
\text { Collection Development in the Health Sciences } \\
\text { Endowment }\end{array}$ & 36,168 & - & - & - & - & 36,168 \\
\hline Janet Doe Lectureship Endowment & 25,859 & - & - & - & - & 25,859 \\
\hline $\begin{array}{l}\text { Carla J. Funk Governmental Relations Award } \\
\text { Endowment }\end{array}$ & 11,115 & 3,025 & - & - & - & 14,140 \\
\hline Eugene Garfield Research Fellowship Endowment & 125,101 & - & - & - & - & 125,101 \\
\hline $\begin{array}{l}\text { T. Mark Hodges International Service Award } \\
\text { Endowment }\end{array}$ & 4,815 & - & - & - & - & 4,815 \\
\hline $\begin{array}{l}\text { Hospital Libraries Section/MLA Professional } \\
\text { Development Grant Endowment }\end{array}$ & 27,680 & - & - & - & - & 27,680 \\
\hline David A. Kronick Traveling Fellowship Endowment & 26,132 & 355 & - & - & - & 26,487 \\
\hline Joseph Leiter NLM/MLA Lectureship Endowment & 41,328 & - & - & - & - & 41,328 \\
\hline $\begin{array}{l}\text { Librarians without Borders }{ }^{\circledR} \text { Ursula Poland International } \\
\text { Scholarship Endowment }\end{array}$ & 22,475 & 40 & - & - & - & 22,515 \\
\hline $\begin{array}{l}\text { Donald A. B. Lindberg Research Fellowship } \\
\text { Endowment }\end{array}$ & 204,762 & 6,400 & - & - & - & 211,162 \\
\hline Majors/MLA Chapter Project of the Year Endowment & 11,500 & - & - & - & - & 11,500 \\
\hline $\begin{array}{l}\text { Lucretia W. McClure MLA Excellence in Education } \\
\text { Award Endowment }\end{array}$ & 33,584 & 1,050 & - & - & - & 34,634 \\
\hline John P. McGovern Award Lectureship Endowment & 115,585 & - & - & - & - & 115,585 \\
\hline Scholarship Endowment & 256,944 & 2,723 & - & - & - & 259,667 \\
\hline Shaping Our Future Endowment & - & - & - & - & - & 48,269 \\
\hline Totals, permanently restricted net assets & $\$ 1,208,458$ & $\$ 16,357$ & - & - & - & $\$ 1,224,815$ \\
\hline Total all net assets & $\$ 3,291,599$ & $\$ 2,856,769$ & $\$(49,970)$ & $\$ 0$ & $\$(2,921,576)$ & $\$ 3,176,822$ \\
\hline
\end{tabular}

Table 1

Continued 